\title{
INFINITELY MANY NONISOMORPHIC NILPOTENT ALGEBRAS
}

\author{
CHONG-YUN CHAO
}

1. Introduction. In [1], the following theorem was proved:

THEOREM A. There exist uncountably many nonisomorphic nilpotent Lie algebras of class $3\left(L \supset L^{2}=[L, L] \supset 0\right)$ over the real number field for any given dimension $\geqq 10$.

The main purpose here is in $\$ 2$, by using a similar method as in $[2$, p. 72$]$, to prove a generalization of Theorem A. Namely, the following

THEOREM 1. Let $K$ be a field whose characteristic is not 2 and whose cardinality is $\boldsymbol{\aleph}_{0}$ (greater than $\boldsymbol{\aleph}_{0}$ ). Then there exist countably infinitely (uncountably) many nonisomorphic nilpotent Lie algebras of class 3 over $K$ for any given dimension $\geqq 10$.

Following from Theorem 1 we can easily show

Theorem 2. Let $K$ be a field whose characteristic is not 2 and whose cardinality is $\boldsymbol{\aleph}_{0}$ (greater than $\boldsymbol{\aleph}_{0}$ ). Then there exist countably infinitely (uncountably) many nonisomorphic solvable not nilpotent Lie algebras of index $3\left(L \supset L^{2} \supset\left[L^{2}, L^{2}\right] \supset 0\right)$ over $K$ for any given dimension $\geqq 11$.

In $\S 3$, we point out that the method used in [1] can also be applied to nilpotent associative algebras.

2. Nilpotent Lie algebras. Let $L$ be a Lie algebra of dimension $m+n$ over a field $F$ defined by a basis $\left(x_{1}, \cdots, x_{m}, y_{1}, \cdots, y_{n}\right)$ with a bilinear antisymmetric bracket multiplication such that

$$
\left[x_{j}, x_{k}\right]=\sum_{i=1}^{n} c_{j k}^{i} y_{i}, \quad j, k=1,2, \cdots, m
$$

where $c_{j k}^{i} \in F$ and not all $c_{j k}^{i}$ are zero and all other products are zero. Then $L$ is a nilpotent Lie algebra of class 3 . The constants $c_{j k}^{i}$ are called the structure constants, and the matrices $C^{(i)}=\left(c_{j k}^{i}\right)$ are called the structure matrices. We assume that the center of $L, Z(L)$, is the commutator $L^{2}=[L, L]$ of $L$ and we determine how the structure matrices change as we shift to another basis $\left(z_{1}, \cdots, z_{m}, z_{m+1}, \cdots\right.$,

Presented to the Society November 29, 1969; received by the editors May 5, 1969. 
$\left.z_{m+n}\right)$ of $L$ with structure constants $d_{j k}^{i}$ in $F, 1 \leqq i, j, k \leqq m+n$. We may assume that $\left(z_{1}, z_{2}, \cdots, z_{m}\right)$ are independent modulo $L^{2}$, i.e., they span a complement $V$ of $L^{2}$ in $L$. We can write $z_{m+i}=v_{i}+t_{i}$ with $v_{i} \in V$ and $t_{i} \in L^{2}$ for $i=1,2, \cdots, n$. Clearly, $\left(z_{1}, \cdots, z_{m}, t_{1}, \cdots, t_{n}\right)$ is still a basis for $L$. Since $\left[z_{i}, z_{j}\right] \in L^{2}$, we have

$$
\left[z_{i}, z_{j}\right]=\sum_{u=1}^{n} d_{i j}^{m+u} t_{u}, \quad i, j=1,2, \cdots, m
$$

and all other products are zero. We note that $\left(x_{1}, x_{2}, \cdots, x_{m}\right)$ also forms a complement $T$ of $L^{2}$ in $L$. Hence, we can replace each $z_{i}$ by an element $s_{i}$ such that $s_{i}-z_{i} \in L^{2}$ and $s_{i} \in T$. Since $L^{2}$ is the center of $L$, the structure constants for the basis $\left(s_{1}, \cdots, s_{m}, t_{1}, \cdots, t_{n}\right)$ are the same as for the basis $\left(z_{1}, \cdots, z_{m}, t_{1}, \cdots, t_{n}\right)$ above.

The set of vectors $\left(s_{1}, \cdots, s_{m}\right)$ is a basis for $T$. Hence, we have $s_{i}=\sum_{p=1}^{m} a_{i p} x_{p}, i=1,2, \cdots, m$ where $A=\left(a_{i p}\right)$ is a nonsingular matrix. Similarly, $y_{r}=\sum_{u=1}^{n} b_{r u} t_{u}, i=1,2, \cdots, n$, where $B=\left(b_{r u}\right)$ is a nonsingular matrix. Then on the one hand $\left[s_{i}, s_{j}\right]=\sum_{u=1}^{n} d_{i j}^{m+u} t_{u}$, and on the other hand

$$
\begin{aligned}
{\left[s_{i}, s_{j}\right] } & =\left[\sum_{p=1}^{m} a_{i p} x_{p}, \sum_{v=1}^{m} a_{j v} x_{v}\right] \\
& =\sum_{p} \sum_{v} a_{i p} a_{j v} \sum_{r} c_{p v}^{r} \sum_{u} b_{r u} t_{u} .
\end{aligned}
$$

By the linear independence of $t_{u}$ 's, we have

$$
d_{i j}^{m+u}=\sum_{p} \sum_{v} \sum_{r} a_{i p} a_{j v} c_{p v}^{r} b_{r u} .
$$

In the form of matrices, we have

$$
D^{(u)}=\sum_{r=1}^{u} b_{r u} A C^{(r)} A^{\prime}, \quad u=1,2, \cdots, n
$$

where $D^{(u)}$ are the structure matrices of $\left(d_{i j}^{m+u}\right)$ and $A^{\prime}$ is the transpose matrix of $A$. In particular, we have

Lemma 1. Let $L$ be a nilpotent Lie algebra of class 3 with dimension $m+n$ over a field $F$. The multiplication of $L$ is defined by (1) such that $Z(L)=L^{2}$. If $M$ is a Lie algebra isomorphic to $L$ then (3) holds where $D^{(u)}, u=1,2, \cdots, n$, are structure matrices of $M$.

Proof. Let $\theta$ be the isomorphic map of $M$ onto $L$. Then $M$ is a nilpotent Lie algebra of class 3 with dimension $m+n$ over $F$ such that 
$Z(M)=M^{2}$. Say, $M=\left(\left(z_{1}^{\prime}, \cdots, z_{m}^{\prime}, t_{1}^{\prime}, \cdots, t_{n}^{\prime}\right)\right)$ with

$$
\left[z_{i}^{\prime}, z_{j}^{\prime}\right]=\sum_{u=1}^{n} d_{i j}^{m+u} t_{u}^{\prime}, \quad i, j=1,2, \cdots, m,
$$

and all other products are zero. Let $z_{i}^{\prime} \theta=z_{i}$ for $i=1,2, \cdots, m$, and $t_{u}^{\prime} \theta=t_{u}$ for $u=1,2, \cdots, n$. Since $\theta$ is an isomorphism of Lie algebras, we obtain (2). Consequently, (3) follows.

Let $K$ be an infinite field whose characteristic is not 2 and let $L_{\alpha}=\left(\left(x_{1}, x_{2}, \cdots, x_{8}, y_{1}, y_{2}\right)\right)$ be an 10 dimensional Lie algebra over a field $K$ defined by

$$
\begin{array}{llrl}
{\left[x_{1}, x_{2}\right]} & =y_{1}+y_{2}, & {\left[x_{3}, x_{4}\right]} & =y_{1}+2 y_{2}, \\
{\left[x_{5}, x_{6}\right]} & =y_{1}, & {\left[x_{7}, x_{8}\right]} & =y_{1}+\alpha y_{2}
\end{array}
$$

where $\alpha \in K, \alpha \neq 0,1,2$ and all other products are zero. Clearly, $L_{\alpha}$ is a nilpotent Lie algebra of class 3 . For convenience, we let $J$ and $C_{\alpha}$ be the structure matrices $C^{(1)}=\left(c_{i j}^{1}\right)$ and $C^{(2)}=\left(c_{i j}^{2}\right)$ of $L_{\alpha}$ respectively, i.e.,

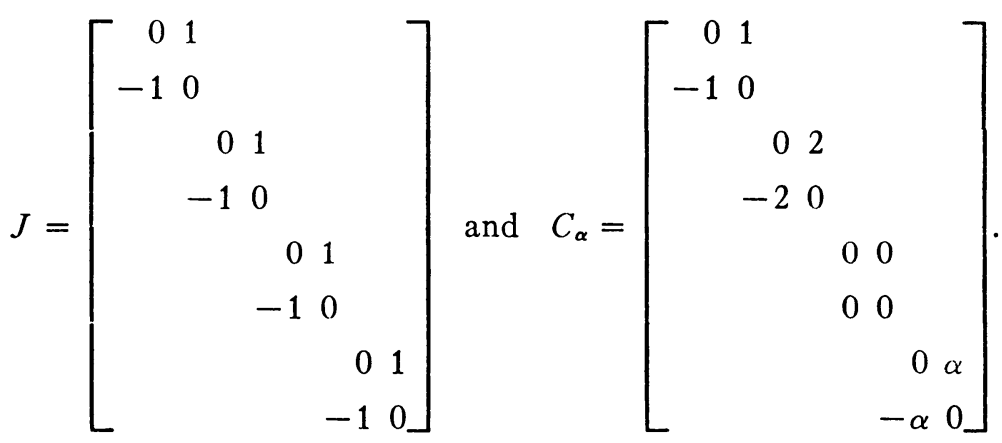

Since $\alpha \neq 0,1,2$, any two different nonzero products are linearly independent, and $L_{\alpha}^{2}$ is generated by $y_{1}$ and $y_{2}$. Let $x \in Z(L)$. Then $x=\sum_{i=1}^{8} a_{i} x_{i}+\sum_{j=1}^{2} b_{j} y_{j}$ and

$$
0=\left[x, x_{k}\right]=\sum_{i=1}^{8} a_{i} \sum_{t=1}^{2} c_{i k}^{t} y_{t} \quad \text { for } k=1,2, \cdots, 8 .
$$

By the linear independence of $y_{t}^{\prime}$ s, we have $\sum_{i=1}^{8} a_{i} c_{i k}^{t}=0$ for $t=1,2$ and $k=1,2, \cdots, 8$. By using $\sum_{i=1}^{8} a_{i} c_{i k}^{1}=0, k=1,2, \cdots, 8$, we have $a_{1}=a_{2}=\cdots=a_{8}=0$. Hence, $x=\sum_{j=1}^{2} b_{j} y_{j} \in L^{2}$ and $Z(L)=L^{2}$.

Lemma 2. Let $\alpha$ be an element of an infinite field $K$ whose characteristic is not 2 such that $\alpha(\alpha-1)(\alpha-2)(3 \alpha-4)(3 \alpha-2) \neq 0$. Then there are 
only finitely many $\beta$ such that $L_{\beta} \cong L_{\alpha}$.

Proof. Suppose $L_{\beta} \cong L_{\alpha}$. Then by (3) we have

$$
\begin{aligned}
J & =b_{11} A J A^{\prime}+b_{21} A C_{\alpha} A^{\prime}, \\
C_{\beta} & =b_{12} A J A^{\prime}+b_{22} A C_{\alpha} A^{\prime} .
\end{aligned}
$$

Multiply both equations by $J^{-1}=-J$ on the left, we obtain:

$$
\begin{aligned}
I & =J^{-1} J=b_{11} J^{-1} A J A^{\prime}+b_{21} J^{-1} A C_{\alpha} A^{\prime}, \\
J^{-1} C_{\beta} & =b_{12} J^{-1} A J A^{\prime}+b_{22} J^{-1} A C_{\alpha} A^{\prime} .
\end{aligned}
$$

Since $I$ and $J^{-1} C_{\beta}$ are diagonal matrices, so are $J^{-1} A J A^{\prime}$ and $J^{-1} A C_{\alpha} A^{\prime}$. Let $d=J^{-1} A J A^{\prime}$. Then

$$
d=\left\{d_{1}, d_{1}, d_{2}, d_{2}, d_{3}, d_{3}, d_{4}, d_{4}\right\}=\frac{1}{|B|}\left(b_{22} I-b_{21} J^{-1} C_{\beta}\right)
$$

where \{\} denotes a diagonal matrix, $|B|$ is the determinant of $B$,

$$
\begin{aligned}
d_{1} & =\frac{1}{|B|}\left(b_{22}-b_{21}\right), & d_{2} & =\frac{1}{|B|}\left(b_{22}-2 b_{21}\right), \\
d_{3} & =\frac{1}{|B|} b_{22} \quad \text { and } & d_{4} & =\frac{1}{|B|}\left(b_{22}-\beta b_{21}\right) .
\end{aligned}
$$

Since $A^{\prime}=J^{-1} A^{-1} J d$ and $J^{-1} A C_{\alpha} A^{\prime}$ is a diagonal matrix, $J^{-1} A C_{\alpha} J^{-1} A^{-1} J d$ is a diagonal matrix. Consequently, $J^{-1} A C_{\alpha} J^{-1} A^{-1} J$ $=\left(J^{-1} A\right)\left(C_{\alpha} J^{-1}\right)\left(J^{-1} A\right)^{-1}$ is a diagonal matrix whose eigenvalues are the same as the eigenvalues of $C_{\alpha} J^{-1}$, namely, $1,1,2,2,0,0, \alpha, \alpha$.

Let $\omega_{1}, \omega_{2}, \cdots, \omega_{8}$ be a permutation of $1,1,2,2,0,0, \alpha, \alpha$. Then (4) and (5) can be written as

$$
\begin{aligned}
& I=b_{11}\left\{d_{1}, \cdots, d_{4}\right\}+b_{21}\left\{\omega_{1}, \cdots, \omega_{8}\right\}\left\{d_{1}, \cdots, d_{4}\right\}, \\
& \{1,1,2,2,0,0, \beta, \beta\} \\
& =b_{12}\left\{d_{1}, \cdots, d_{4}\right\}+b_{22}\left\{\omega_{1}, \cdots, \omega_{8}\right\}\left\{d_{1}, \cdots, d_{4}\right\} .
\end{aligned}
$$

Since $d=J^{-1} A J A^{\prime}$ and $J$ and $A$ are nonsingular, $d$ is nonsingular. Hence, $b_{22} \neq 0$. Write the matrix equation ( 7$)$ as 8 equations, and using $b_{22} \neq 0$, we have $\omega_{1}=\omega_{2}, \omega_{3}=\omega_{4}, \omega_{5}=\omega_{6}$ and $\omega_{7}=\omega_{8}$. Then we have 4 possible cases:

Case 1. $J^{-1} A C_{\alpha} J^{-1} A^{-1} J=\left\{0,0 . \omega_{4}, \omega_{4}, \omega_{6}, \omega_{6}, \omega_{8}, \omega_{8}\right\}$,

Case 2. $J^{-1} A C_{\alpha} J^{-1} A^{-1} J=\left\{\omega_{4}, \omega_{4}, 0,0, \omega_{6}, \omega_{6}, \omega_{8}, \omega_{8}\right\}$,

Case 3. $J^{-1} A C_{\alpha} J^{-1} A^{-1} J=\left\{\omega_{4}, \omega_{4}, \omega_{6}, \omega_{6}, 0,0, \omega_{8}, \omega_{8}\right\}$,

Case 4. $J^{-1} A C_{\alpha} J^{-1} A^{-1} J=\left\{\omega_{4}, \omega_{4}, \omega_{6}, \omega_{6}, \omega_{8}, \omega_{8}, 0,0\right\}$

where $\omega_{4}, \omega_{6}, \omega_{8}$ is a permutation of $1,2, \alpha$. 
Now we consider the Case 1: The matrix equation (6) can be written as:

$$
\begin{aligned}
& 1=b_{11} d_{1}, \\
& 1=b_{11} d_{2}+b_{21} \omega_{4} d_{2}, \\
& 1=b_{11} d_{3}+b_{21} \omega_{6} d_{3}, \\
& 1=b_{11} d_{4}+b_{21} \omega_{8} d_{4} .
\end{aligned}
$$

Also, the matrix equation ( 7$)$ can be written as:

$$
\begin{aligned}
& 1=b_{12} d_{1}, \\
& 2=b_{12} d_{2}+b_{22} \omega_{4} d_{2}, \\
& 0=b_{12} d_{3}+b_{22} \omega_{6} d_{3}, \\
& \beta=b_{12} d_{4}+b_{22} \omega_{8} d_{4} .
\end{aligned}
$$

We may assume $d_{1}=1$. The reason is that the equations (4) and (5) still hold if we replace $A$ by $(\lambda A)$ and $B$ by $\left(\lambda^{-2} B\right)$ where $\lambda \neq 0$ and $\lambda$ belongs to $K$ or $\lambda$ belongs to an extension field of $K$. We have

$$
\begin{aligned}
I & =b_{11}^{\prime} J^{-1} E J E^{\prime}+b_{21}^{\prime} J^{-1} E C_{\alpha} E^{\prime}, \\
J^{-1} C_{\beta} & =b_{12}^{\prime} J^{-1} E J E^{\prime}+b_{22}^{\prime} J^{-1} E C_{\alpha} E^{\prime}
\end{aligned}
$$

where $b_{i j}^{\prime}=b_{i j} \lambda^{-2}, i, j=1,2$ and $E=(\lambda A)$. We choose $\lambda^{2}$ to be $|B| /$ $\left(b_{22}-b_{21}\right)$, we have

$$
\begin{array}{r}
J^{-1} E J E^{\prime}=\frac{1}{|\bar{B}|}\left(b_{22}^{\prime} I-b_{21}^{\prime} J^{-1} C_{\beta}\right)=\{1,1, \cdots\} \\
\text { where }|\bar{B}|=b_{11}^{\prime} b_{22}^{\prime}-b_{12}^{\prime} b_{21}^{\prime} .
\end{array}
$$

Hence, we may assume $d_{1}=1$.

From (8) and (12), we have $d_{1}=b_{11}=b_{12}=1$. By (14), $b_{22}=-1 / \omega_{6}$. By (13), we have

$$
d_{2}=2 \omega_{6} /\left(\omega_{6}-\omega_{4}\right)
$$

By (9), we have

$$
b_{21}=-\left(\omega_{6}+\omega_{4}\right) / 2 \omega_{4} \omega_{6} .
$$

By (10), we have

$$
d_{3}=2 \omega_{4} /\left(\omega_{4}-\omega_{6}\right) .
$$

By (11), we have

$$
d_{4}=2 \omega_{4} \omega_{6} /\left(2 \omega_{4} \omega_{6}-\omega_{6} \omega_{8}-\omega_{4} \omega_{8}\right) .
$$


Substitute into (15), we obtain

$$
\beta=2 \omega_{4}\left(\omega_{6}-\omega_{8}\right) /\left(2 \omega_{4} \omega_{6}-\omega_{6} \omega_{8}-\omega_{4} \omega_{8}\right) .
$$

Since $\alpha$ satisfies the condition in the lemma, $2 \omega_{4} \omega_{6}-\omega_{6} \omega_{8}-\omega_{4} \omega_{8} \neq 0$.

In the following cases, we may still assume $d_{1}=1$. By using similar computations as in the Case 1 , we obtain:

In Case $2, \beta=2 \omega_{4}\left(\omega_{8}-\omega_{6}\right) /\left(-\omega_{4} \omega_{6}+2 \omega_{4} \omega_{8}-\omega_{6} \omega_{8}\right)$.

In Case 3, $\beta=2 \omega_{8}\left(\omega_{4}-\omega_{6}\right) /\left(-\omega_{4} \omega_{6}+2 \omega_{4} \omega_{8}-\omega_{6} \omega_{8}\right)$.

In Case $4, \beta=2 \omega_{8}\left(\omega_{6}-\omega_{4}\right) /\left(-\omega_{4} \omega_{6}-\omega_{4} \omega_{8}+2 \omega_{6} \omega_{8}\right)$.

Hence, there are only finitely many $\beta$ such that $L_{\beta} \cong L_{\alpha}$. In fact, for each $\alpha$, the possible $\beta$ 's are:

$$
\alpha,-(\alpha-2), \frac{2 \alpha}{3 \alpha-2}, \frac{4-2 \alpha}{4-3 \alpha}, \frac{4-4 \alpha}{4-3 \alpha} \text { and } \frac{4 \alpha-4}{3 \alpha-2} .
$$

Now the proof of Theorem 1 goes as follows: Let $K^{\prime}$ be the set consisting of all the elements of $K$ except $0,1,2,4 / 3,2 / 3$ if the characteristic of $K$ is not 3. If the characteristic of $K$ is 3 , let $K^{\prime}$ be the set consisting of all the elements of $K$ except $0,1,2$. Then the cardinality of $K^{\prime}$ is still $\boldsymbol{\aleph}_{0}$ (greater than $\boldsymbol{\aleph}_{0}$ ). For the given dimension $n=10$, on $K^{\prime}$ we define a relation $\alpha \sim \beta$ if and only if $L_{\alpha} \cong L_{\beta}$ where $L_{\alpha}$ and $L_{\beta}$ are nilpotent Lie algebras of dimension 10 over $K$ as constructed in Lemma 2. Clearly, this relation is an equivalence relation. Hence, $K^{\prime}$ is partitioned into countably infinitely (uncountably) many pairwise disjoint classes since by Lemma 2 each class consists of only a finite number of elements. The nilpotent Lie algebras corresponding to any two different classes are nonisomorphic. Hence, there are countably infinitely (uncountably) many nonisomorphic nilpotent Lie algebras of class 3 and of dimension 10 over $K$.

For $n>10$, let $n=10+m$ and construct the direct sum of nilpotent Lie algebras $L_{\alpha}{ }^{\prime}=L_{\alpha} \oplus M$ where $M$ is an abelian Lie algebra of dimension $m$, and $L_{\alpha}$ is the nilpotent Lie algebra of dimension 10 as constructed in Lemma 2. Then $L_{\alpha}^{\prime} \cong L_{\beta}^{\prime}$ if and only if $L_{\alpha} \cong L_{\beta}$. Theorem 1 follows.

Now the proof of Theorem 2: Let $L_{\alpha}=\left(\left(x_{1}, \cdots, x_{8}, y_{1}, y_{2}\right)\right)$ be defined as in Lemma 2 , let $N_{\alpha}=\left(\left(x_{1}, \cdots, x_{8}, y_{1}, y_{2}, z_{\alpha}\right)\right)$ where the multiplications of $x_{i}{ }^{\prime}$ s and $y_{j}{ }^{\prime}$ 's are defined the same as in $L_{\alpha}$, and $\left[z_{\alpha}, x_{i}\right]=x_{i}, i=1,2, \cdots, 8$, and $\left[z_{\alpha}, y_{j}\right]=2 y_{j}, j=1,2$. Then $N_{\alpha}$ is a solvable not nilpotent Lie algebra of dimension 11 over $K . N_{\alpha} \cong N_{\beta}$ if and only if $L_{\alpha} \cong L_{\beta}$. By Theorem 1, there are countably infinitely (uncountably) many nonisomorphic solvable not nilpotent Lie algebras of dimension 11 over $K$. 
For any given dimension $n^{\prime}>11$, let $n^{\prime}=11+m$ and construct the direct sum of solvable Lie algebras $N_{\alpha}^{\prime}=N_{\alpha} \oplus M$ where $M$ is an abelian Lie algebra of dimension $m$. Then $N_{\alpha}^{\prime} \cong N_{\beta}^{\prime}$ if and only if $N_{\alpha} \cong N_{\beta}$ and Theorem 2 follows.

A field $R$ is said to be an $S$-field ( $S^{\prime}$-field) if $R$ contains a set $S\left(S^{\prime}\right)$ of countably infinitely (uncountably) many elements which are algebraically independent over a subfield $Q$ of $R$. Clearly, an $S^{\prime}$-field is also an $S$-field. Although Theorem A proved in [1] is over the real number field, it still holds if the real number field is replaced by an $S^{\prime}$-field. In addition, we have the following statement: There exist countably infinitely many nonisomorphic nilpotent Lie algebras of class 3 over an $S$-field for any given dimension $n \geqq 10$. The proof is practically the same as the one in [1]. Certainly, this statement is an immediate consequence of Theorem 1 . However, the nilpotent Lie algebras constructed in the proofs are different; in the former case, the center always coincides with the commutator. In the later case, when the dimension of the Lie algebra is odd, the center properly contains the commutator.

3. Nilpotent associative algebras. In [2, Theorem 16, p. 72] it is shown that for any integer $n \geqq 6$, there exist infinitely many nonisomorphic commutative nilpotent associative algebras of class 3 and of dimension $n$ over an infinite field $F$. It seems natural to ask whether there exist infinitely many nonisomorphic nilpotent associative algebras of dimension lower than 6 . The method used in [2] does not seem to work when the dimension is lower than 6 . Since a nilpotent Lie algebra of class 3 is an associative algebra, we may apply the method used in [1] to prove the following:

THEOREM 3. Let $F$ be an $S$-field ( $S^{\prime}$-field) then there exist countably infinitely (uncountably) many nonisomorphic nilpotent associative algebras of class 3 over $F$ for any given dimension $\geqq 5$.

Let $U$ be a subfield of a field $R$. An associative algebra $\mathfrak{A}$ over $R$ is said to be an $U$-algebra if its structure constants with respect to some basis of $\mathfrak{A}$ lie in $U$.

Let $E$ be a subfield of $F$ and $\mathfrak{A}$ be an associative algebra of dimension $m+n$ over $F$ defined by a basis $\left(x_{1}, \cdots, x_{m}, y_{1}, \cdots, y_{n}\right)$ with a bilinear multiplication $x_{j} x_{k}=\sum_{i=1}^{n} c_{j \mathrm{k}}^{i} y_{i}, j, k=1,2, \cdots, m$, and all other products are zero where $c_{j k}^{i} \in F$. Then $\mathfrak{A}$ is a nilpotent associative algebra of class 3 .

LeмmA 3. If the numbers $c_{j k}^{i}, 1 \leqq i \leqq n, 1 \leqq j, k \leqq m$ are algebraically independent over $E$, and if $m n^{2}>n^{2}+m^{2}$, then $\mathfrak{A}$ is not an E-algebra. 
We omit the proofs of Lemma 3 and Theorem 3 here since they are practically the same as the proofs in [1]. Although by Theorem 16 in [2] we only need to prove for the case of $\mathfrak{A}$ having dimension 5 , (i.e., $m=3$ and $n=2$ ) our construction shows that the annihilator of $\mathfrak{A}$ coincides with $\mathfrak{A}^{2}$, and $\mathfrak{A}$ is not commutative.

In particular, we have

COROLlary. There exist uncountably many nonisomorphic nilpotent associative algebras of class 3 over the real or complex number field for any given dimension $\geqq 5$.

\section{REFERENCES}

1. C. Y. Chao, Uncountably many nonisomorphic nilpotent Lie algebras, Proc. Amer. Math. Soc. 13 (1962), 903-906. MR 26 \#6221.

2. D. A. Suprunenko and R. I. Tškevič, Commutative matrices, Nauka i Tehnika Press, Minsk, 1966; English transl., Academic Press, New York, 1968. MR 34 \#1356.

University of Pittsburgh 